ISSN 1997-5902

\title{
Distribution des Glossines vecteurs de la Trypanosomose humaine africaine dans le Parc National de Moukalaba Doudou (Sud-ouest Gabon).
}

\author{
Ely S. DIBAKOU1* ${ }^{*}$, Franck MOUNIOKO ${ }^{1}$, Christophe R. ZINGA KOUMBA ${ }^{2,3}$, Ornella A. MBANG \\ NGUEMA ${ }^{1}$, Geneviève ACAPOVI-YAO ${ }^{4}$, JacquesF. MAVOUNGOU1,2 \\ 1 Université des Sciences et Techniques de MASUKU, BP 941, Franceville, Gabon ; \\ 2 Institut de Recherche en Ecologie Tropicale (IRET), BP 13354, Libreville, Gabon ; \\ ${ }^{3}$ Ecole Régionale Post-Universitaire d'Aménagement et de gestion Intégrés des Forêts et Territoires Tropicaux \\ (ERAIFT), BP 15373, Kinshasa, R. D. Congo ; \\ 4 Université Félix Houphouët - Boigny, UFR Biosciences 22, BP : 582 ; Abidjan 22, Côte d'Ivoire ; \\ *Auteur correspondant : DIBAKOU Ely Serge, email : sergeely@yahoo.fr
}

Original submitted in on 23rd January 2015. Published online at www.m.elewa.org on 28 ${ }^{\text {th }}$ February 2015 http://dx.doi.org/10.4314/jab.v86i1.7

\section{RESUME}

Objectifs : Dans le but de connaitre la densité apparente et la composition spécifique des glossines dans un ancien foyer de la Trypanosome humaine africaine au Gabon, une enquête entomologique a été menée dans deux biotopes du Parc National de Moukalaba Doudou (Sud Gabon) : le village Doussala et la forêt attenant à ce village.

Méthodologie et résultats : Les captures ont été effectuées à l'aide de pièges Vavoua placés en forêt et au village Doussala durant la grande saison sèche. Au total, 4071 glossines ont été capturées : $3141(77,2 \%)$ l'ont été en forêt et $930(22,8 \%)$ au village. En forêt, six espèces ont été capturées avec des abondances différentes : $\mathrm{G}$. fuscipes fuscipes (DAP=11,1 G/P/J), G. nashi (DAP=0,49 G/P/J), G. frezili $(D A P=0,25 \mathrm{G} / \mathrm{P} / \mathrm{J})$, G. fusca congolensis (DAP=0,18 G/P/J), G. palpalis palpalis (DAP=0,09 G/P/J) et $\mathrm{G}$. tachinoides (DAP=0,01 $G / P / J)$. Tandis qu'au village, cinq espèces ont été identifiés, il s'agit de $G$. fuscipes fuscipes qui a une DAP de 3,48 G/P/J. Les autres espèces à savoir $G$. tachinoides, G. palpalis palpalis, G. nashi et $G$ frezili ont des densités apparentes respectivement de $0,06 \mathrm{G} / \mathrm{P} / \mathrm{J} ; 0,02 \mathrm{G} / \mathrm{P} / \mathrm{J} ; 0,01 \mathrm{G} / \mathrm{P} / \mathrm{J}$ et $0,01 \mathrm{G} / \mathrm{P} / \mathrm{J}$.

Conclusion et application: Tous les éléments du complexe pathogène nécessaire à la transmission de la Trypanosome humaine africaine sont présents dans ce parc. Ces résultats bien que préliminaires invitent à élaborer des stratégies de lutte et de contrôle des vecteurs de parasitoses.

Mots clés : piège Vavoua, Doussala, Trypanosome, Glossines, Gabon.

ABSTRACT:

Distribution of biological vectors of human African Trypanosomiasis in the National Park of Moukalaba Doudou (South Gabon).

Objective: In order to know the apparent density and specific composition of the flies in a former home of human African trypanosomes in Gabon, an entomological investigation was conducted in primary forest and Doussala village in the National Park of Moukalaba Doudou (Gabon South). 


\section{Dibakou et al. J. Appl. Biosci. Distribution des Glossines vecteurs de la Trypanosomose humaine}

africaine dans le Parc National de Moukalaba Doudou (Sud-ouest Gabon).

Methodology and Results:The catches were taken with the Vavoua traps placed in primary forest and the village Doussala during the long dry season. A total of 4071 tsetse with 3141 (77.2\%) in the forest and 930 $(22.8 \%)$ in the village were captured. In the forest, sixs pecies were captured with different abundances $G$. fuscipes fuscipes (DAP=11.1 G/ P/J), G. nashi (DAP=0.49 G/ P/J), G frezili (DAP=0.25 G / P /J), G fusca congolensis (DAP=0.18 G / P /J), G palpalis palpalis $(\mathrm{DAP}=0.09 \mathrm{G} / \mathrm{P} / \mathrm{J}$ ) and $\mathrm{G}$ tachinoides $(\mathrm{DAP}=0.01 \mathrm{G} / \mathrm{P}$ /J).While in the town five species have been identified, it is $G$. fuscipes fuscipes DAP which has a $3.48 \mathrm{G} / \mathrm{P} / \mathrm{J}$. Other species namely $G$ tachinoides, $G$ palpalis palpalis, $G$. nashi and $G$ frezili have apparent densities of 0.06 G / P /J, respectively; 0.02 G / P /J; $0.01 \mathrm{G} / \mathrm{P} / \mathrm{J}$ and $0.01 \mathrm{G} / \mathrm{P} / \mathrm{J}$.

Conclusion and Application:All elements of the complex pathogenic necessary for the transmission of human African trypanosomes are present in the park. These results, although preliminary invite to develop strategies of struggle and control parasitosis vectors.

Keywords: Vavoua trap, Doussala, Trypanosoma, Glossina, Gabon.

\section{INTRODUCTION}

La trypanosomose humaine africaine ou maladie du sommeil est une affection parasitaire due à la présence dans le sang, la lymphe et lou le liquide céphalo-rachidien d'un protozoaire flagellé sanguicole exoerynthrocytaire appelé trypanosome, du genre Trypanosoma sp (Aksoy, 2003 ; Mbida et al., 2009, Peka et al., 2014). Diverses espèces du genre Glossina, mâles et femelles, en assure la transmission à l'homme (Aksoy, 2003, Courtin et al., 2005 ; Mbida et al., 2009; Bosson et al., 2012). Et actuellement, environ 60 millions de personnes sont exposées et on estime de 300 à 500000 celles qui sont atteintes (OMS, 1998). Par ailleurs, la THA est à l'origine à elle seule, dans de nombreux pays d'Afrique centrale, de $87 \%$ du nombre total de malades diagnostiqués dans toute l'Afrique (WHO, 2006). De plus, les maladies à transmission vectorielle figurent parmi les principales causes de morbidité et de mortalité pour les hommes et les animaux. Par conséquent, leurs impacts sur la santé humaine sont considérables (WHO, 2006). Au Gabon, les connaissances portant sur la THA et en particulier sur ses vecteurs demeurent encore mal connues et fragmentaires. En effet, de nombreux foyers historiques sont connus dans plusieurs provinces, mais ces foyers n'ont pas été prospectés depuis plus de 15 ans en raison de l'insuffisance de moyens financiers dont dispose le programme national de lutte contre la trypanosomiase humaine africaine de ce pays (Kohagne et al., 2011). Pourtant, des études récentes conduites dans le foyer historique de la THA de Bendjé (Gabon) ont montré la présence des glossines infectées de trypanosomes (Kohagne et al., 2011). Par ailleurs, des cas sporadiques de THA sont diagnostiqués de manière passive par les structures sanitaires de la ville de port Gentil dans la province de l'Ogooué maritime (Amblard, 1996). La province de la Nyanga, ancien foyer de la THA et attenant à celle de l'Ogooué maritime, abrite le Parc National de Moukalaba Doudou. Aussi, ce parc regorge une faune particulière représentée par les grands mammifères comme les éléphants (Loxodonta africana cyclotis), les buffles (Syncerus caffer nanus), les gorilles (Gorilla g gorilla), les chimpanzés (Pan troglodytes) et les céphalophes (Cephalophus $s p p$.). La fréquentation régulière de ce milieu par ces animaux fait de ce parc un espace écotouristique intéressant et très prisé par les touristes. De plus, avec la mise en exécution du projet Conservation de la Biodiversité en Forêt Tropicale à travers la Coexistence Durable entre l'Homme et l'Animal (PROCOBHA) dans ce parc, les contacts entre populations humaines et animales seront facilités avec la venue importante de touristes mais aussi des populations locales. Finalement, l'une des conséquences possibles de ces contacts sera une augmentation de la probabilité de rencontre hôtevecteur et donc des échanges de pathogènes entre l'Homme, la faune et le milieu (Epstein et al., 1993). En outre, les risques sanitaires vont probablement s'accroitre et la fréquence d'apparition de nouveaux cas de THA pourraient augmenter au fur et à mesure que les populations humaines seront plus 
nombreuses à multiplier les contacts avec cet environnement. C'est dans ce cadre qu'une étude entomologique basée sur l'inventaire des glossines a été menée dans deux biotopes caractéristiques du

\section{MATÉRIEL ET MÉTHODES}

Zone d'étude: L'étude a été menée dans le Parc National de Moukalaba-Doudou situé dans la partie sudouest du Gabon. Ce parc couvre une superficie de 5028 $\mathrm{km}^{2}$ (figure 1). II comprend plusieurs types d'habitats dont les plus importants sont: les milieux humides, les savanes, les forêts, les formations rocheuses et les grottes. Le milieu anthropisé est constitué par le village Doussala. Par ailleurs, le paysage floristique de la région comprend de nombreuses espèces qu'on ne voit pas ou rarement ailleurs au Gabon (Vande, 2012). La rivière Moukalaba constitue le principal affluent de la Nyanga. Elle serpente dans une plaine couverte d'une mosaïque de forêts et de savanes. La faune sauvage est représentée par plusieurs espèces de grands mammifères dont les buffles (Syncerus caffer nanus), les éléphants (Loxodonta africana cyclotis), les chimpanzés (Pan troglodytes), les gorilles (Gorilla gorilla) et les céphalophes (Cephalophus spp.). L'avifaune comprend plusieurs espèces dont euplecte monseigneur (Euplectes hordeaceus) (Vande, 2012). Le climat de cette région est de type équatorial caractérisé par une saison sèche de quatre à cinq mois, de mai à septembre; et une saison des pluies de sept à huit mois, allant d'octobre à avril ou mai pratiquement ininterrompue (Takenoshita et al., 2008 ; Ebang et Juichi, 2014). La pluviométrie annuelle moyenne du parc est de $2000 \mathrm{~mm}$ de précipitations dans l'extrême nord et seulement $1600 \mathrm{~mm}$ dans le sud. Les températures varient en fonction des saisons. La différence entre les mois les plus frais et les mois les plus chauds (mars et avril) est de l'ordre de 3 à $4{ }^{\circ} \mathrm{C}$. La différence entre la température journalière maximale et minimale augmente avec l'altitude. Capture et identification des Glossines : L'enquête entomologique s'est déroulée du 17 juillet au 22 août 2014 en grande saison sèche. Les glossines ont été capturées à l'aide de
PNMD. L'objectif de cette étude a été de déterminer les densités apparentes des glossines dans la forêt primaire et au village Doussala attenant à la forêt primaire.

pièges Vavoua (Laveissière et Grebaut, 1990). Ce dernier est un piège monoconique composé d'une cage de capture ,d'un piquet central (en bois ou en fer de béton), d'un cône en tulle moustiquaire et de trois écrans (surface attractive) cousu à $120^{\circ}$. Ces trois écrans sont composés d'une bande bleue (bleu phtalogène) externe et d'une bande noire centrale. Ces écrans sont maintenus en place par un fil de fer galvanisé. La partie supérieure est constituée de trois angles arrondis à leur base, cousus ensemble. Le piège est fixé au sol soit par un piquet en bois ou soit par un fer à béton, soit encore par un bambou. L'attractivité visuelle est le principe de ce piège. Elle dépend surtout de la longueur d'onde du rayonnement réfléchi par la couleur bleue et le noir. Le bleu phtalogène attire l'insecte et le noir favorise la pose de ce celui-ci. Ces pièges ont été posés dans deux types de biotopes identifiés pour l'étude à savoir la forêt primaire et village Doussala ou milieu anthropisé. Dans chaque biotope, un dispositif de 7 pièges Vavoua distants l'un de l'autre d'au moins $500 \mathrm{~m}$ a été utilisé. Les relevés quotidiens des glossines ont été faits à $7 \mathrm{~h} 00$ durant 37 jours successifs par biotope prospecté. Les glossines collectées ont été ainsi introduites dans les cages munis d'étiquettes portant l'heure de collecte, le numéro du piège et le milieu de capture. Les insectes collectés ont été ramenés au Laboratoire d'Écologie Vectorielle (LEVIRET). Au laboratoire, les glossines ont été triées et séparées des autres insectes, puis dénombrés et identifiés sous une loupe binoculaire à l'aide des clés d'identification publiées par Brunhes et al (1998) et Pollock, 1992. La forme des forcipules inférieurs nous a permis de distinguer Glossina palpalis palpalis de Glossina fuscipes fuscipes. 

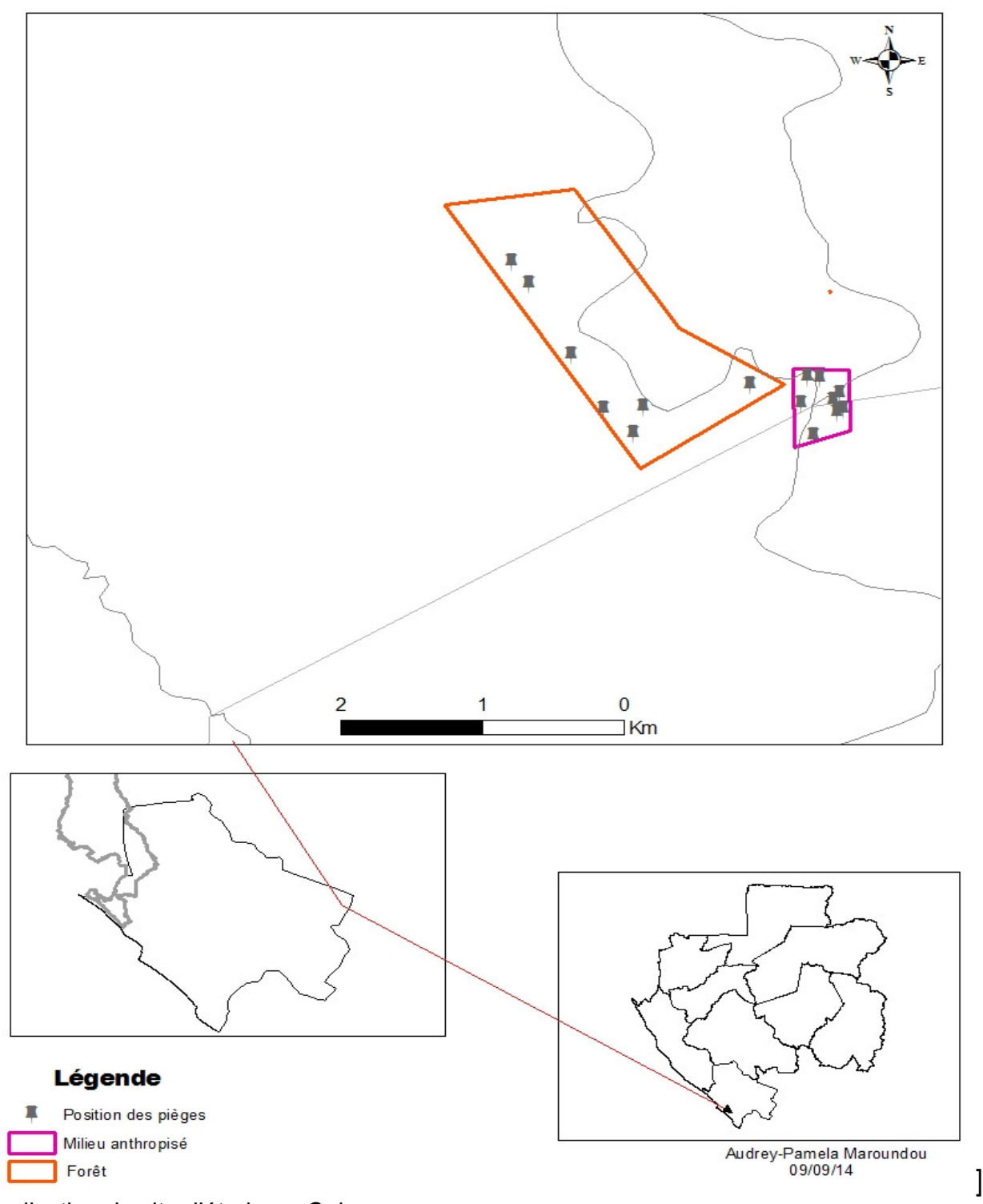

Figure 1 : Localisation du site d'étude au Gabon

Analyse des données : L'abondance de chaque espèce des Glossines capturées a été définie par la densité apparente par piège et par jour (DAP) calculée selon la formule suivante :

$D A P=\frac{\text { Nombre de mouchescapturées }}{\text { Nombrede piègesx nombrede joursde capture }}$
La diversité spécifique des glossines dans les milieux explorés a été déterminée par l'indice de diversité de Shannon, qui permet de quantifier l'hétérogénéité de la biodiversité d'un milieu. Cet indice a été calculé à l'aide de la formule suivante :

$H^{\prime}=-\Sigma(\mathrm{Ni} / \mathrm{N}) \times \log (\mathrm{Ni} / \mathrm{N})$

Avec Ni le nombre d'individus d'une espèce donnée et $\mathrm{N}$ le nombre total d'individus.

glossines dont $3141(77,2 \%)$ en forêt et $930(22,8 \%)$ au village (milieu anthropisé). Le plus grand nombre de

\section{RÉSULTATS}

L'enquête entomologique réalisée dans le Parc National de Moukalaba Doudou a permis d'identifier 4071 


\section{Dibakou et al. J. Appl. Biosci. Distribution des Glossines vecteurs de la Trypanosomose humaine}

africaine dans le Parc National de Moukalaba Doudou (Sud-ouest Gabon).

captures a été observé en forêt. Les densités apparentes par piège et par jour (DAP) ont été de 12,12 G/P/J en forêt et de 3,59 G/P/J au village. Parmi les glossines capturées en forêt, nous avons dénombré 2192 femelles
$(69,8 \%)$ et 949 mâles $(30,2 \%)$ soit un sex ratio (F/M) de 2,31. Tandis qu'au village 664 femelles (72\%) ont été capturées contre 266 mâles (28\%) soit un sex ratio (F/M) de 2,49 (figure 2).

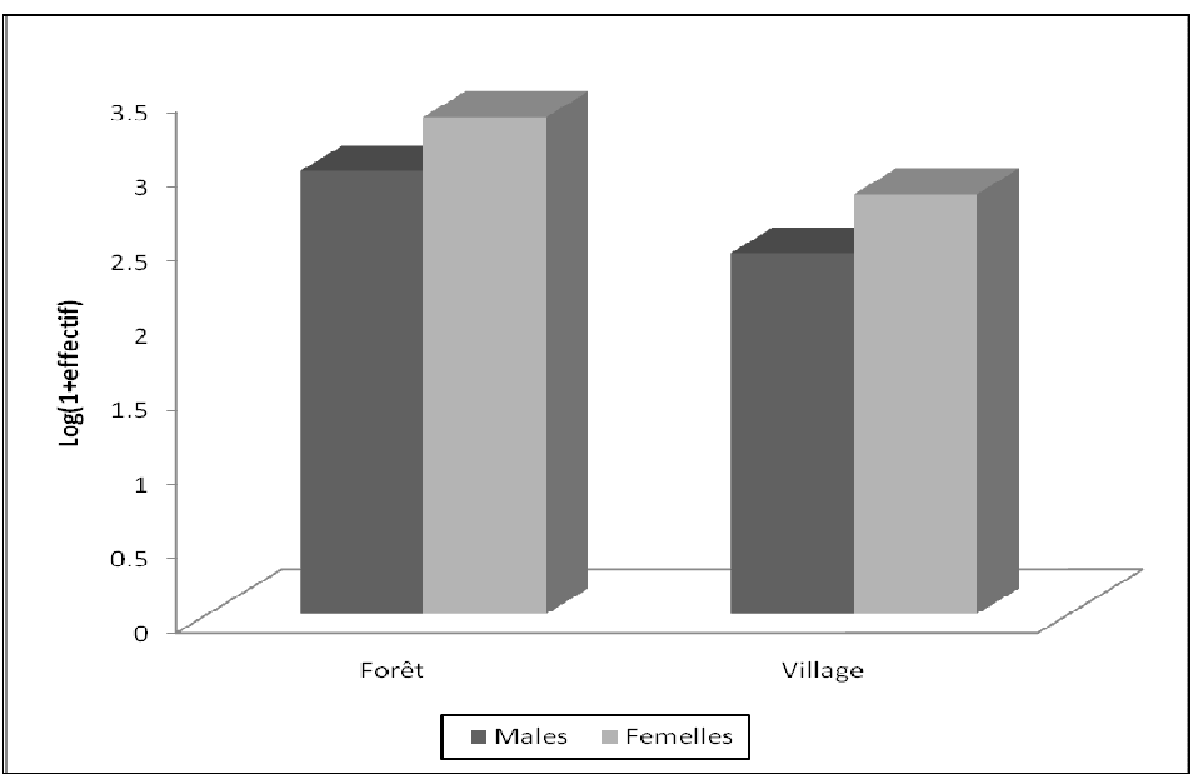

Figure 2 : Répartition des mâles et femelles de glossines en fonction du biotope.

Composition et diversité spécifique des glossines dans le PNMD : Au total six espèces de glossines ont été capturées dans le PNMD avec des abondances variables. En effet, Glossina fuscipes fuscipes a été l'espèce la plus abondante ( $30 \%$ ) suivit de Glossina nashi
$(18 \%)$. Les autres espèces à savoir Glossina frezili (15\%), Glossina fusca congolensis (14\%), Glossina palpalis palpalis (12\%) et Glossina tachinoides (11\%) ont été moins bien représentées (figure 3).

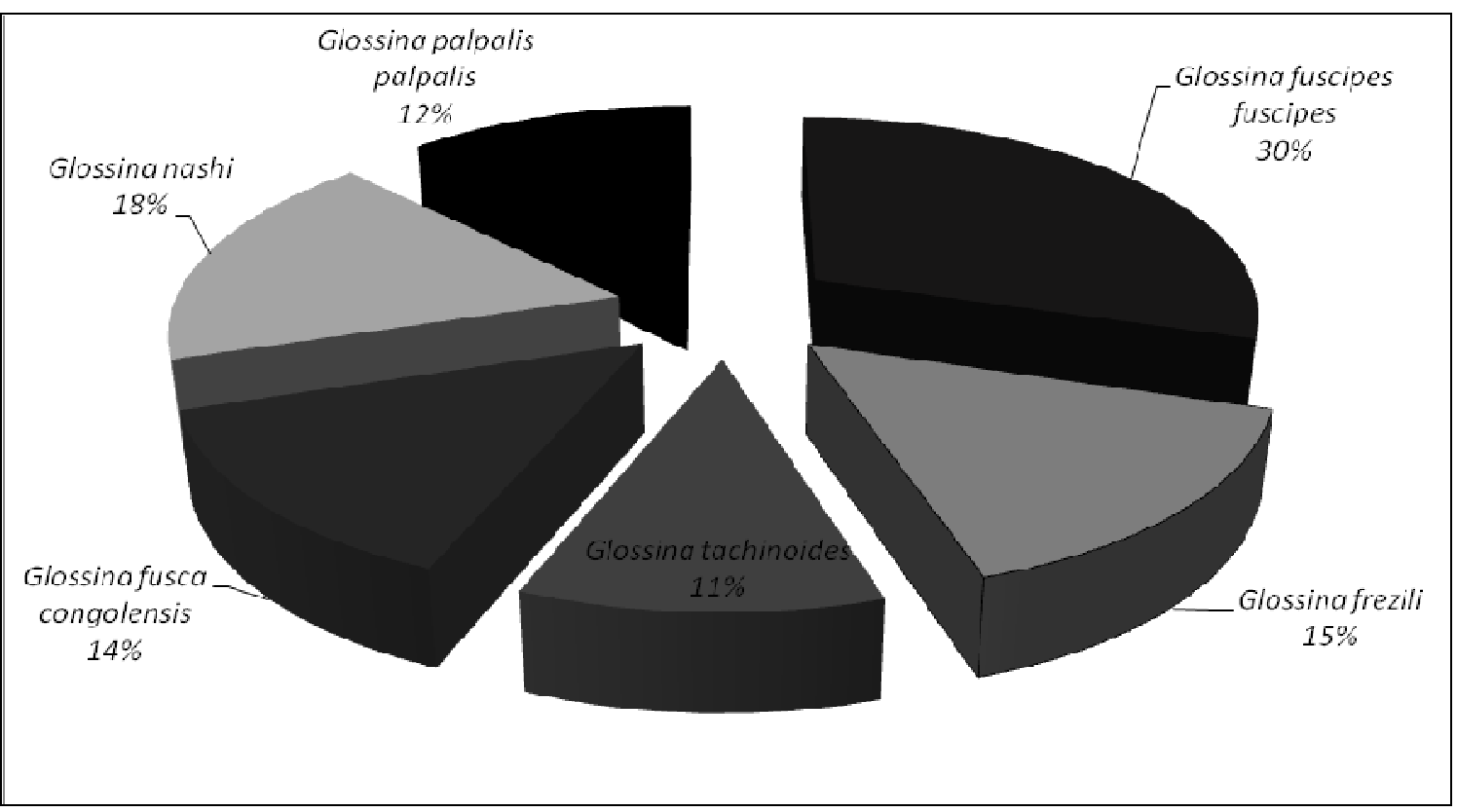




\section{Dibakou et al. J. Appl. Biosci. Distribution des Glossines vecteurs de la Trypanosomose humaine africaine dans le Parc National de Moukalaba Doudou (Sud-ouest Gabon).}

Figure 3 : Répartition des Glossines capturées dans le Parc National de Moukalaba Doudou

\begin{abstract}
Abondance des glossines en fonction des biotopes prospectés : La diversité du peuplement des glossines dans les deux biotopes prospectés a été évaluée par l'indice de diversité de Shannon ( $\left.H^{\prime}\right)$. Ces indices ont été respectivement de 0,87 en forêt et 0,43 en milieu anthropisé. En forêt, six espèces ont été identifiées : $G$. fuscipes fuscipes a été l'espèce la mieux représentée avec $91,6 \%$. Les autres espèces ont été très faiblement capturées: $G$. nashi $(4,1 \%), G$ frezili $(2,1 \%), G$ fusca congolensis $(1,5 \%), G$ palpalis palpalis $(0,73 \%)$ et $G$ tachinoides $(0,1 \%)$. Aussi, ces insectes ont présenté des densités variables dont la plus élevée a été observée chez $\mathrm{G}$. fuscipes fuscipes (DAP=11,1 G/P/J). Les quatre autres espèces ont présenté des très faibles DAP : $G$. nashi (DAP=0,49 G/P/J), G frezili (DAP=0,25 G/P/J), $G$
\end{abstract}

\section{DISCUSSION}

Les résultats obtenus dans cette étude ont montré clairement que la forêt attenant au village Doussala et ce dernier ont un fond entomologique commun en matière de glossines. Et ce fond entomologique commun serait lié au fait que les deux milieux subissent les mêmes facteurs écologiques (climat, présence d'hôtes nourriciers, etc.). En effet, l'infestation d'un milieu par les glossines est conditionnée par la présence simultanée des facteurs environnementaux adéquats (température comprise entre $15^{\circ} \mathrm{C}$ et $25^{\circ} \mathrm{C}$, luminosité, humidité relative) et des vertébrés hôtes nourriciers (Darchen, 1978, Foil \& Gorham, 2000, Solano et al., 2010). Aussi, ce résultat suggère que malgré l'activité anthropique (élevage, agriculture etc.) dans le village Doussala qui modifie considérablement ce paysage, la biodiversité glossinienne n'est pas affectée de manière considérable. Cependant, l'abondance et la distribution de ces insectes n'a pas été uniforme et varie selon les biotopes prospectés. L'abondance et la diversité des glossines ont été plus importantes en forêt qu'en milieu anthropisé représenté par le village Doussala. Cette répartition pourrait s'expliquer par la différenciation des paysages et à la structure des milieux pouvant engendrer des microhabitats particuliers plus ou moins favorables au développement des glossines. Aussi, la conjugaison de plusieurs facteurs tels que le climat, une végétation dense, la présence d'hôtes nourriciers, seraient à l'origine de la forte densité de glossines dans une zone donnée (Frezil \& Carnevale, 1976 ; Laveissière \& Hervouët, 1981; Gouteux \& Kiénou, 1982 ; Zinga et al., 2013, 2014). Ainsi, de telles conditions sembleraient se fusca congolensis ( $\mathrm{DAP}=0,18 \mathrm{G} / \mathrm{P} / \mathrm{J}), \mathrm{G}$ palpalis palpalis (DAP=0,09 G/P/J) et $\mathrm{G}$ tachinoides (DAP=0,01 G/P/J). Au village, on a noté la présence de cinq espèces: $G$. fuscipes fuscipes $(97 \%), G$ tachinoides $(1,7 \%), G$. palpalis palpalis $(0,6 \%)$, $G$. nashi $(0,3 \%)$ et $G$ frezili $(0,3 \%)$. Aussi, ces espèces ont des densités apparentes très faibles à l'exception de $G$. fuscipes fuscipes qui a une $D A P$ de $3,48 \mathrm{G} / \mathrm{P} / \mathrm{J}$. Les autres espèces à savoir $G$. tachinoides, G. palpalis palpalis, G. nashi et $G$. frezili ont respectivement $0,06 \mathrm{G} / \mathrm{P} / \mathrm{J} ; 0,02 \mathrm{G} / \mathrm{P} / \mathrm{J} ; 0,01 \mathrm{G} / \mathrm{P} / \mathrm{J}$ et $0,01 \mathrm{G} / \mathrm{P} / \mathrm{J}$. Toutes les espèces de glossines capturées au village ont aussi été capturées en forêt mais avec des abondances différentes. Seule G. fusca congolensis n'a pas été capturée au village.

retrouver dans la forêt et expliqueraient les fortes densités des glossines qui y sont observées. La faible richesse spécifique observée dans les milieux prospectés pourrait s'expliquer par le fait qu'un seul type de piège a été utilisé et que seulement 14 pièges soit 7 points de capture par biotope ont été explorés pendant la grande saison sèche. De ce fait, ces résultats d'inventaire ne peuvent être exhaustifs. D'autres captures sont nécessaires avec plusieurs types de pièges et d'attractifs, dans un plus grand nombre de sites et sur une période plus longue, pour avoir une image plus précise de la richesse spécifique des glossines dans le PNMD. En effet, des études antérieures ont montré que l'association de plusieurs types de piège et l'ajout d'un attractif augmenteraient considérablement les captures d'insectes hématophages (Djieteye, 1992, Amsler \& Filledier, 1994, Zinga et al., $2013 ; 2014)$. Aussi, notre étude a été conduite en grande saison sèche, période pendant laquelle les glossines riveraines se dispersent peu et restent confinées dans des habitats aux conditions environnementales favorables à leur survie (Bouyer, 2009 ; Mbida, 2009; Peka et al., 2014). Cette faible dispersion pourrait justifier probablement les densités spécifiques des glossines capturées dans le PNMD. Par ailleurs, les femelles ont été plus nombreuses que les mâles; ce qui pourrait s'expliquer par le fait que leur longévité est plus grande que celle des mâles (Laveissière, 2000). Les femelles ont besoin absolument du repas de sang pour la maturité des larves et par conséquent elles auront un temps de vol plus important que celui des mâles. Ces résultats sont similaires à ceux 
obtenus par Bosson et al. (2012) en Côte d'lvoire. L'abondance de $G$ fuscipes fuscipes et les faibles captures de G. palpalis palpalis dans les deux biotopes prospectés serait probablement le résultat de la compétition interspécifique entre ces deux espèces qui a déjà été démontré au Cameroun et au Congo (Mouchet et al., 1958 ; Laveissière et al., 1997). Par ailleurs, la présence de G. palpalis palpalis dans ce parc concorde avec l'ancienne carte de distribution des glossines au

\section{CONCLUSION}

Comme les résultats le démontrent, cette étude a permis de mettre en évidence une liste non exhaustive de la biodiversité glossinienne dans le PNMD. Cette liste pourra servir de référence aux études complémentaires qui seront effectuées pour comprendre l'impact de la saison sur la dynamique spatio-temporelle des glossines. Les différences de densité et de composition spécifique des glossines observées entre biotopes prospectés émanent de la différenciation des paysages pouvant engendrer des conditions plus ou moins favorables au développement des Glossines dans cet ancien foyer de la THA. La présence d'une part des animaux domestiques

\section{REMERCIEMENTS}

Ce travail a été réalisé grâce à l'appui institutionnel, financier et logistique de l'Institut de Recherche en Écologie Tropicale (IRET-CENAREST), l'Université des Sciences et Techniques de MASUKU (USTM), le Laboratoire d'Écologie Vectorielle (LEV-IRET), le Projet pour la Conservation De La Biodiversité En forêt Tropicale A Travers La Coexistence Durable Entre L' Homme Et L'Animal (PROCOBAH), l'École Régionale Post-Universitaire d'Aménagement et de Gestion Intégrés des Forêts et Territoires Tropicaux (ERAIFT-UNIKIN) et

\section{BIBLIOGRAPHIE}

Aksoy .S., 2003. Control of tsetse flies and trypanosomes using molecular genetics. Vet Prasitol., 115 : 125-145.

Amblard, J., 1996. Situation de la trypanosomiase humaine africaine au Gabon. Etude sur I période 1993-1995. Rapport interne du service des grandes endémies, Ministère de la santé, Gabon, 18p

Amsler,S., Filledier, J., 1994. Attractivité pour les Tabanidae de l'association métacrésol/octénol : Résultats obtenus au Burkina Faso. Revue Elevage et de Médecine vétérinaire des Pays Tropicaux., 47 : 93-96.
Gabon qui montre une large distribution de cette sous espèce (Maillot, 1953). Ainsi, sa présence, bien que faible témoigne de son ubiquité pour des milieux dont les températures sont comprises entre $20^{\circ} \mathrm{C}$ et $25^{\circ} \mathrm{C}$ (Schweiz, 1915). La très faible présence de $G$. nashi, $G$ frezili, $G$ fusca congolensis et $G$ tachinoides dans les biotopes prospectés traduit leur exigence du point de vue écologique. Ces résultats sont similaires à ceux obtenus par Mbida et al. (2009).

et sauvages, réservoirs potentiels de Trypanosomes ; et d'autre part des Glossines en particulier de $G$ fuscipes fuscipes et $G$ palpalis palpalis vecteurs majeurs des Trypanosomes Humaines et Animales, pourraient entretenir la survie des Trypanosomes dans cet ancien foyer. Aussi, tous les éléments du complexe pathogène nécessaire à la transmission de la THA et de la TAA sont présents dans ce parc et pourrait présumer une possible indemnisation de cette parasitose. Ces résultats bien que préliminaires invitent à élaborer des stratégies de lutte et de contrôle des vecteurs de parasitoses.

la CEEAC/PACEBO. Nous remercions LOGNO NZABA Roye pour son aide technique sur le terrain et Madame MAROUNDOU Audrey Pamela pour la réalisation de la carte. Aussi nous remercions Mlle BELL Rayna Camille pour la traduction du résumé en anglais. Nos sincères remerciements sont dirigés à l'adresse de Mr. Ephrem NZENGUE, Mr. Clency MIKALA, Mr. Karl HENGA BOTSIKA BOBE, Mr. Nathaniel KOUMBA et Mr. Patrick ONDO OKAMA dont les remarques, suggestions et corrections ont permis d'améliorer ce manuscrit.

Bosson-Vanga, A.H., Acapovi-Yao, G., Kaba, D., Dofini, F., Coulibaly, B., N'dri Louis., Kone, M., 2012. Infection de Glossina palpalis palpalis par les trypanosomes le long du fleuve Comoé dans la région d'ABENGOUROU (côte d'Ivoire). J. Sci. Pharm. Biol., 3(1) : 31-37.

Bouyer, J., 2009. La dispersion des glossines. Insectes., 21.

Brunhes, J., Cuisance, D., Geoffroy, B., Hervy, J.P., 1998. Les glossines ou mouches tsé-tsé. Logiciel d'identification et d'enseignement. Eds ORSTOM, Montpellier, France. 


\section{Dibakou et al. J. Appl. Biosci. Distribution des Glossines vecteurs de la Trypanosomose humaine africaine dans le Parc National de Moukalaba Doudou (Sud-ouest Gabon).}

Courtin, F., Dupont, S., Zeze, D.G., Jamonneau, V., Sané, B., Coulibaly, B., Cuny, G., Solano. P., 2005. Trypanosomose Humaine Africaine: Transmission urbaine dans le foyer de Bonon (Côte d'Ivoire).Tropical Medecine and International Health., 10(4) : 340-346.

Darchen, R., 1978. Les populations d'Agelena consociate Denis, araignée sociale, dans la forêt primaire gabonaise. Leur répartition et leur densité. Annales de sciences naturelles, Zoologie, Paris, $14(2): 19-26$.

Djiteye, A., 1994.Efficacité comparée des différents types de pièges et / ou associations d'odeurs sur les tabanides, stomoxes et glossines présentes en zone soudano-guinéenne (Mali) G.m. submorsitans, G. tachinoides, G. p. gambiensis. Résumé Rapport d'étape, LCV de Bamako (Mali).

Ebang Ella, G.W., Juichi Yamagiwa., 2014.Use of tool sets by chimpanzees for multiple purposes in Moukalaba-Doudou National Park, Gabon. Primates,6pp. DOI 10.1007/s10329-014-0431-5.

Epstein, P.R., Ford, T.E., Colwell R.R., 1993. Marine ecosystems: emerging diseases as indicators of change. Lancet.,342: 1217-1219.

Foil, L.D., Gorham, J.R., 2000. Mechanical transmission of disease agents by arthropods. In: B.F. Eldridge and J.D. Edman, Medical Entomology. Dordrecht, the Netherlands, Kluwer Academic Publishers, 461-514.

Frezil, J.L., Carnevale, P., 1976. Utilisation de la carboglace pour la capture de Glossina fuscipes quanzensis Pires, 1948, avec le piège ChallierLaveissière. Conséquences épidémiologiques. Cahiers ORSTOM, Série Entomologie Médicale et Parasitologie, $14: 225$ - 233.

Gouteux, J.P., Kiénou, J.P., 1982. Observations sur les glossines d'un foyer forestier de trypanosomiase humaine en Côte d'Ivoire. 5. Peuplement de quelques biotopes caractéristiques: Plantations, forêts et galeries forestières, en saison des pluies. Cahiers ORSTOM, Série Entomologie Médicale et Parasitologie, 20: 41-61.

Laveissière, C., Hervouët, J.P., 1981. Population de glossines et occupation de l'espace. Enquête entomologique préliminaire dans la région de la Lobo, (Côte d'Ivoire). Cahiers ORSTOM, série Entomologie Médicale et Parasitologie, 19 : 247260.
Lavessière, C., Grébaut., P. 1990. The trapping of tsetse flies (Diptera: Glossinidae). Improvement of a model: the Vavoua trap. Tropical Medicine and Parasitology, 41 (2): 185-192.

Laveissière, C., Sané, B., Diallo, P.B., Truc, P., MEDA, A.H., 1997. Le risque épidémiologique dans un foyer de la maladie du sommeil en Côte d'Ivoire. Tropical Medicine \& International Health, 8 : 729-732.

Laveissière, C., Grébaut, P., Herder, S., et al., 2000. Les glossines vectrices de la Trypanosomiase humaine. OCEAC ed., 246P.

Mbida Mbida, J.A., Mimpfoundi, R., Njiokou, F., Manga, L., Laveissière C., 2009. Distribution et écologie des vecteurs de la trypanosomose humaine africaine de type savanicole en zone de forêt dégradée au sud Cameroun : cas du foyer de Doumé. Bulletin de la Société Pathologique Exotique., 102 : 101-105.

Maillot, C.L., 1953. Répartition des Glossines en A. E. F. Bulletin de la Société de Pathologie Exotique., 46 (2): 195-197.

Mouchet, J., Gariou, J., Rateau J., 1958. Distribution géographique et écologique de Glossina palpalis palpalis ROB. - DESV. Et Glossina fuscipes fuscipes Newst. Au Cameroun. Bulletin de Société de Pathologie Exotique., 51 : 652-661.

OMS., 1998. Control and surveillance of African Trypanosomiasis. Report of a WHO Expert Committee, WHO Technical Report Series 881.

Peka Mallaye., Kohagne Tongué, L., Ndeledje, N., Louis,F.J., Mahamat Hassane, H., 2014. Transmission concomitante de trypanosome humaine et animale : le foyer de Mandoul au Tchad. Revue d'élevage et de médecine vétérinaire des pays tropicaux, 67(1) : 5-12

Pollock, J.N., 1992. Manuel de lutte contre la mouche Tsé-tsé. Volume 1 : Biologie, systématique et répartition des tsé-tsé. F.A.O; Rome, 310 p.

Schwetz, J., 1915. Preliminary note on the general distribution of Glossina palpalis in Lomani district, Belgian, Congo. Annals of Tropical Medecine and Parasitology., 9: 513-526.

Solano, P., Bouyer, J., Itard, J., Cuisance, D., 2010. The cyclical vectors of trypanosomes. Infectious and para diseases of livestock., 13: 155-183.

Takenoshita, Y., Ando, C., Iwata, Y., Yamagiwa, J., 2008. Fruit phenology of the great habitat in the Moukalaba-Doudou National Park, Gabon. 
African Study Monograph Supplementary., 39: 23-39.

Vande, W.J.P., 2012. MOUKALABA DOUDOU. Agence National des Parcs Nationaux (ANPN), Libreville, Gabon. 296 p.

Who-World Health Organization., 2006. Weekly epidemiological record-Relevé épidémiologique hebdomadaire, 81 (8): 69-80.

Zinga Koumba, C.R., Bouyer J., Mavoungou, J.F., Acapovi-Yao,G.L., KohagneTongué,L., Mbang Nguema, O.A., Obame Ondo, K.P., Mutambwe,S., 2013. Évaluation de la diversité des diptères hématophages dans une clairière marécageuse du Gabon à l'aide des pièges Vavoua et Nzi.Revue Elevage et de Médecine Vétérinaire des Pays Tropicaux.,66 : 91-96.

Zinga Koumba, C.R., Mbang Nguema, O.A., Kohagne, T.L., Acapovi Yao, G.L., Obame, O.K.P., Mutambwe, S., Mavoungou, J.F., 2014. Contribution à l'évaluation de la diversité des vecteurs biologiques de la Trypanosomose Humaine Africaine et de leur activité journalière dans le Parc National de I'Ivindo (Nord est Gabon). Journal of Applied Biosciences, 80:7060-7070. 